\title{
Artifact Based Geometric Constructions
}

\author{
Kalliopi Siopi \\ University of Patras \\ Eugenia Koleza \\ University of Patras
}

\begin{abstract}
This study examines the integration of an artifact in teaching Euclidean geometry at upper secondary school, as a way of enabling students to enter the theoretical world of a geometrical proof. The key assumption underlying this research was that the deliberate use of artifacts in mathematics instruction may render detectable learning processes. In particular, we worked with students who had never worked with geometric tools before, apart from pair of compasses and rulers, observing and analyzing their geometric reasoning, while they performed geometric constructions using the artifact. Results demonstrate that the physical, and functional characteristics of the artifact, and the type of mathematics it incorporates, allows students to reach geometric conclusions by induction, based on the mathematics of the artifact, and devise ways, based on the mathematics of the artifact, to prove their initial reasoning.
\end{abstract}

Keywords: artifact, instrumental genesis, Euclidean geometry

\section{INTRODUCTION}

According to Trninic \& Abrahamson (2012, p.283) "artifacts are ready-to-hand cultural equipment created by "packaging" procedures for skillfully encountering particular situations, intrigue scholars of human cognition and development for - at least - three reasons: (1) philosophically, artifacts constitute essential cultural objects to think-and-act-with; (2) pedagogically, education involves learning to use the tools humanity has found indispensable; and (3) methodologically, artifacts render learning processes more visible for investigative scrutiny". Nevertheless, it is a usual practice in teaching geometry, to have students prove statements or theorems given by the teacher or the textbook in an abstract way, solely based on other existing axioms or theorems. Because of this, many students have difficulties in constructing and understanding justifications and proofs in geometry. A 'solution' to this problem comes from Archimedes. In a letter addressed to Erathostenes, he described his "mechanical method" which allows someone to treat mathematical questions with mechanical considerations. Thus, the tool, via the mechanical method allows the transformation of the physical experience

in formal models. But this transformation is not obvious. Mariotti (2002) taking as an example the construction of a circle by using the pair of compasses, comments that for learners the passage from the use of the pair of compasses to trace circular shapes to the conception of the circle as 'the locus of the points equidistant from the centre' is not immediate. Jore and Parzysz (2005, in Fujita, Jones, Kunimune, 2013, 3 10) refer to a 'twilight zone' between the practical aspects of physically carrying out a geometrical 
construction and the theoretical aspect of proof. They conclude (p.121) that giving too much precision in the description of the gestures to be performed to make a 'construction' may have as consequence that some students will remain quite a long time in a 'geometry of drawings' and that it is worth investigating ways to enable students "to move towards a 'theoretical geometry'. It is of great importance through teaching to make students understand that any geometrical construction corresponds to a theorem, which means that there is a proof that validates the construction procedure that solves the corresponding construction problem" (Mariotti, 2013, p.444). For the Theory of Semiotic Mediation (TSM) unfolding the semiotic potential of an artifact is a starting point of a didactic process viewing the entry of students to the theoretical world of geometry. Bussi and Mariotti (2008), referring to the artifact's semiotic potential, distinguish between personal meanings - constructed meanings arising in the individual from using the artifact as a means of accomplishing a prescribed task- and mathematical meanings - meanings that an expert recognizes as mathematical when observing the student's use of the artifact-. "By taking advantage of its semiotic affordance, the teacher utilizes the artifact to occasion opportunities for students to develop personal meaning into mathematical meanings that constitute the didactical goals." (Trninic et al, 2010, p.1525). But, how may it happen that a student relates practices with the artefact to mathematics? ...how may it happen that personal meanings arising in the accomplishment of a task through the use of a certain artefact may become mathematical meanings? (Mariotti, 2013, p.442). Research, with specific kinds of artifacts, in the context of TSM offers an answer to this question.

In our research we are interested in the way students proceed from discovering the potentialities of a simple mechanical linkage, to the creation of geometric constructions and the formulation of proofs. Our research question was: Are students who have never used geometric tools before, apart from the pair of compasses and rulers, (i) capable of discovering the mathematics incorporated in the structure of a new tool and creating geometric constructions, formulations and proofs of theorems; (ii) capable of relating the Mathematics of the tool to their already acquired knowledge of Mathematics?

\section{THE ARTIFACT}

The artifact, which we discuss in this study, is a simple in its complexity 'mathematical machine'. It is a linkage of two rods connected by a joint, one bar having double the length of the other. The rod $\mathrm{AB}$ is connected to the midpoint of the rod $\mathrm{DC}$ at $\mathrm{B}$ and $\mathrm{AB}=\mathrm{CB}=\mathrm{BD}$ (Figure 1). This is first described in the book 'De Organica Conicarum Sectionum Constructione' of Schooten (1646) and appears in Japanese textbooks from 1943 (approved by Monbusyo) for students of 14 years with the aim of realizing mathematization with open-ended approach from the situation to elementary geometry and from elementary geometry to analytic geometry (Bussi, Taimina \& Isoda, 2010). The artifact in question is handy to use, and its manipulation does not demand particular dexterity.

\section{FIGURE 1 THE LINKAGE}

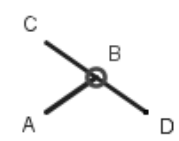

\section{The Potentialities and Constrains the Artifact}

Highlighting the limitations/restrictions and the potentialities of an artifact is necessary in order to become evident the geometric knowledge that integrates his structure. The geometry incorporated in the artifact determines the design of teaching situations and emerges during processing by the user so as to elicit practical and utilitarian transformations of his environment (Vérillon \& Andreucci, 2006).

The structural characteristics of the physical model of the artifact influence and restrict its movement in a particular area of the plane. The hinge/ joint allows the transformation of the form of the artifact without 
making changes in lengths and the relationships between them. When one of the free ends is fixed and the other end (leader-point) follows a path, then it forces the joint to move in a circular arc, while the third end (tracing-point) moves along the plane, tracing a trajectory, the form of which depends on the particular restrictions of the movement of the point-driver (see cases of the trajectories in Table 1).

The hinge brings students face to face with the physical limitations of the artifact (the local feature of the transformation), which induces peculiar utilization schemes (Bartolini Bussi \& Maschietto, 2008). For instance, when the distance of points $\mathrm{A}$ and $\mathrm{D}$ (or A and C) tends to zero, then the form of the assembly degenerates into a line segment of constant length DC.

TABLE 1

TRAJECTORIES OF THE JOINT-POINT B AND OF THE TRACING-POINT C

Case 1. When a point $\mathrm{A}$ is stabilized and a point $\mathrm{D}$
(leader-point) moves on a straight line $\mathrm{AD}$, then the point
$\mathrm{B}$ traces a circle arc with center $\mathrm{A}$ and radius $\mathrm{AB}$, and
point $\mathrm{C}$ traces a line $\mathrm{CA}$ perpendicular to the line $\mathrm{AD}$.
Case 2. When a point $\mathrm{D}$ is stabilized and a point $\mathrm{A}$
(leader-point) moves on a straight line $\mathrm{AD}$, then point $\mathrm{B}$
traces a circle arc with center $\mathrm{D}$ and radius $\mathrm{DB}$ and point
$\mathrm{C}$ traces a circle arc with center $\mathrm{D}$ and radius $\mathrm{DC}$.

In case the leader-point D moves in a circle (with end A steady), what changes is that the tracing-point $\mathrm{C}$ traces a curve whose form depends on the length of the radius and distance between the circle center from the steady point $\mathrm{A}$, while its axis of symmetry is the perpendicular to the diacentric line AD at point $\mathrm{A}$ (see examples of curves of the tracing-point $\mathrm{C}$ in Table 2).

TABLE 2

EXAMPLES OF CURVES OF THE TRACING-POINT C WHEN THE LEADER-POINT C MOVES IN A CIRCLE

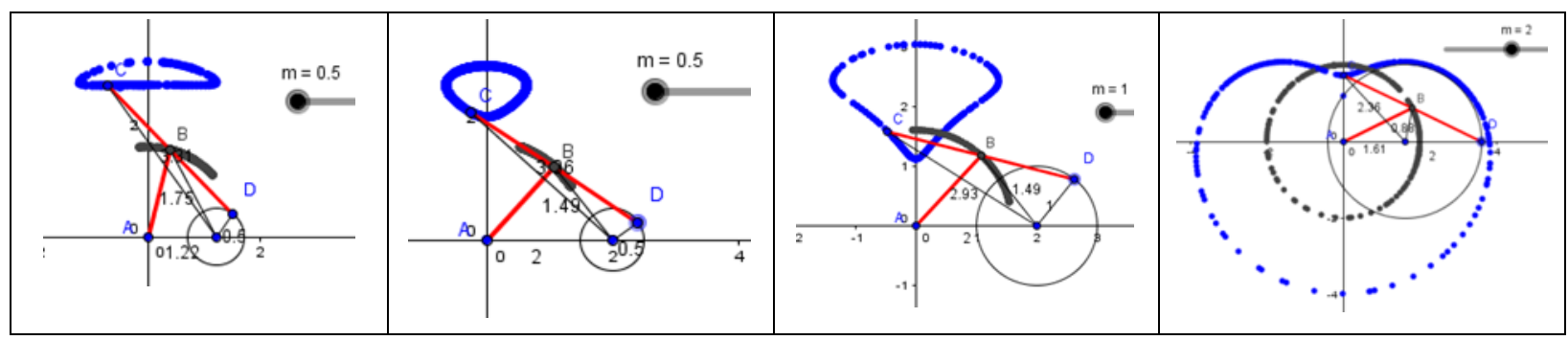

Each one of the aforementioned tool's motion capabilities offers the possibility of exploring a rich range of mathematical tasks. In this study we focus on the transformations resulting from the movement of the leader-point on a straight line.

\section{METHODOLOGY}

Our research was conducted at an upper secondary school. It is a part of a series of teaching experiments in progress, through which we try to investigate whether the intentional use of an artifact -apart from the ruler and the pair of compasses- can activate learning processes in the context of teaching of Euclidean 
geometry in a class of upper secondary school. The teaching experiment was aimed at the observation and analysis of the way students, without any prior experience in using this specific tool exploit the structure and the function of the tool in order to formulate geometric propositions developing arguments and proof strategies. The 'teaching experiment' technique utilized by mathematics education researchers may shed more light on how students' concepts change and are influenced by various instructional methods (Steffe \& Thomposn, 2000).

In our case, we wanted to shed light on the first steps of the instrumental genesis (Vérillon \& Rabardel, 1995) by recording and analyzing students' procedures and thoughts during the instrumentalisation process (Martignone, 2011). Students' exploration processes linked to the study of the artifact components (What does the machine consist of?), the genesis and development of its utilization schemes -the procedures linked to the use of artifacts and its eventual inherent constraints. (What does the machine do?)-Unit 1- and the analysis of the mathematical meanings embodied in the machine (Why does it do that?)-Unit 2.

The sample consisted of 25 students of 10th grade class (15-16 years old), of different learning abilities and interests, who participated voluntarily. All the participating students had no previous experience with any artifact except for the pair of compasses and rulers. In this teaching experiment, 2 two-hour lessons were designed and taught (2015). Each lesson was recorded and the team kept detailed field notes. Students who participated in the experiment worked in 6 groups, 5 groups of 4 people and 1 group of 5 people. The groups were selected to be homogeneous in terms of their cognitive level, verified according to their grades in mathematics. The group of five students consisted of two girls and three boys. Students in this group, for a better team function offered and took the initiative to work as two subgroups on the basis of their gender: one subgroup of 2 girls (S1, S2) and another one of 3 boys (S3, S4, S5). They committed to cooperate at subgroup level and to exchange ideas with all of the core team members. During the experiment, self constructed models of the specific artifact (a part of a climbing plants grid on a plastic platform $(50 \mathrm{~cm}$ $\mathrm{x} 50 \mathrm{~cm}$ ), together with paper, ruler, pencils and nails, were used. Transcripts, visual material (photographs), written reports of the groups and personal notes of the teacher constitute the data of this study. At the beginning of the teaching session, the teacher only set the subject of the investigation Her interventions were limited to the coordination of group discussions. Students worked together in their groups, discussing their solutions and presenting their evidence.

\section{DATA ANALYSIS AND DISCUSSION}

\section{First Unit: What Does the Machine Consist of and Which Are the Mathematical Meanings of the Machine?}

The artifact was displayed by the teacher to the groups without any explanation or information. The students were invited to carefully observe it and explore how it had been constructed in order to analyze the physical structure of the linkage. They observed that the hinge enables the rotation of the two plastic rods, one rod being independent from the other. They utilized this rotation of the rods, turned the small rod and placed it on the bigger one. They repeated this procedure in the opposite direction. This led them to the conclusion that the bigger rod is double the length of the smaller one and that the two rods join at the of the bigger one. Students from both groups identified and described elements of the assembly using clear geometrical terms: they referred to a) "points" for the three rods ends and the position of the hinge, b) line segments for the two rods, and c) angles (with rods as the sides with a common vertex (the hinge), for which they determined their relative geometric relationship -due to their position- as adjacent and supplementary. In their explanations they used axioms (two points determine the position of a straight line), the definition of angle and propositions (two adjacent angles that have their non-common sides as opposite-facing rays are supplementary).

\section{Second Unit: What Does the Machine Do and Why?}

\section{Episode 1- Construction of the Circle}

The girls' group "read" the artifact as a means of constructing circles. To verify their hypothesis, they stabilized on a plastic platform with nails both: the free end $[\Gamma]$ of the smaller rod $[\mathrm{M} \Gamma]$ and the hinge $[\mathrm{M}]$. 
Then, they put a pencil at the two free ends $[\mathrm{A}],[\mathrm{B}]$ of the large rod $[\mathrm{AB}]$ and they began to rotate using as a rotation center the nail on the hinge $[\mathrm{M}]$. Having observed that $\mathrm{MA}=\mathrm{MB}=\mathrm{M} \Gamma$, they hypothesized that points $[\mathrm{A}],[\mathrm{B}]$ and $[\Gamma]$ are points of the same circle. To verify, they removed the nail from the free end $[\Gamma]$ of the small rod $[\mathrm{M} \Gamma]$, they placed on it a third pencil and they observed the trace left as the small $\operatorname{rod}[\mathrm{M} \Gamma]$ turned around.

In essence, from the first moment the students saw behind the artifact a form of a pair of compasses (Table 3).

S1: This is axis [she places her finger vertically on hinge $M$ ] and the others [she means rods $\mathrm{AB}$ and $\mathrm{M} \Gamma$ ] can move circularly ... these [she shows $\mathrm{A}, \mathrm{B}$ and $\Gamma$ ] are moving on a circle.

S2: Let's say this is a centre [points at hinge M] ... and let's place the pencil here ... or here $\ldots$ or here $[$ she puts the pencils on point $\mathrm{A}$ or $\mathrm{B}$ or $\Gamma]$...then a circle has been made.

TABLE 3

EPISODE 1: THE ARTIFACT AS A PAIR OF COMPASSES

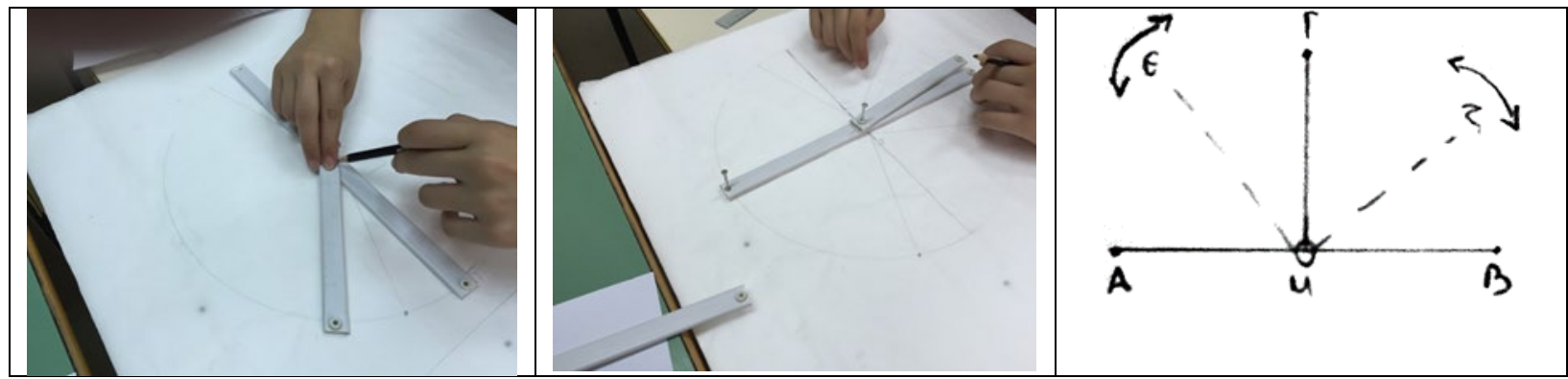

The boys team worked in the same manner as the girls but left free the end of the small rod $[\Delta \Gamma]$ to begin with. They chose to stabilize with nails the ends $[\mathrm{A}],[\mathrm{B}]$ of the large $\operatorname{rod}[\mathrm{AB}]$ on a plastic platform and utilize the rotation capability provided by the hinge $[\Delta]$ on the small $\operatorname{rod}[\Delta \Gamma]$, having placed a pencil at the free end $[\Gamma]$. Observing the trace of the pencil $(\Gamma)$, they formulated the conjecture that the free end $[\Gamma]$ of the small rod $[\Delta \Gamma]$ moves in a half-circle with the large rod $[\mathrm{AB}]$ as diameter, and the hinge $[\Delta]$ as the center. Both teams identified in the various positions of free ends of the two rods, during their turning around the hinge, the quality of points of a circle.

S5: This is stable [the segment AB] ... it has the nail here and here [A and $\mathrm{B}]$ and this $[\Gamma]$ turns ... it leaves marks... which have equal distance from it [points to hinge $\Delta$ ] ... distance equal to $\Delta \Gamma$.

Afterwards, the boys group drew on the paper a straight line $[\varepsilon]$. They stabilized the end $[\mathrm{B}]$ of the large rod $[\mathrm{AB}]$ on a straight line $(\varepsilon)$ and the second free end $[\mathrm{A}]$ on a plastic platform with nails, so that the large rod $[\mathrm{AB}]$ looks perpendicular to the straight line (e). They attempted to place the free end $[\Gamma]$ of the small $\operatorname{rod}[\Delta \Gamma]$ upon the straight line $(\varepsilon)$, without ruining the perpendicularity of the large rod $[A B]$, so as to drag it along the straight line $(\varepsilon)$. A fact which proved impossible.

\section{S3: The small doesn't reach.}

They removed the nail from the upper end $[\mathrm{A}]$ of the large rod $[\mathrm{AB}]$ and bent it at an angle, succeeding in this way in the free end $[\Gamma]$ of the small $\operatorname{rod}[\Delta \Gamma]$ touching the line segment $(\varepsilon)$. They placed the pencil at 
the hinge $[\Delta]$ and at the free end $[\mathrm{A}]$ of the large rod. Dragging free end $[\Gamma]$ on $(\varepsilon)$, they traced two circle arcs. Their drawing was similar to the one in Case 2 on Table 1.

S4: We made a double pair of compasses (table 4).

TABLE 4

EPISODE 1: THE ARTIFACT AS A DOUBLE PAIR OF COMPASSES

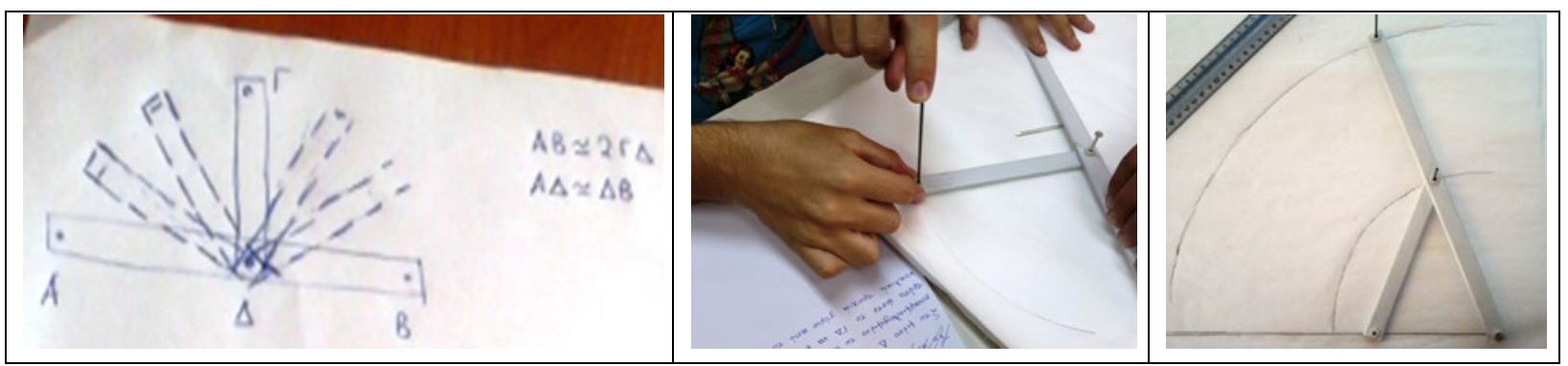

Episode 2- Construction of Right Angles

The girls noticed that the four points [A, B, $\Gamma, \mathrm{M}]$ define two isosceles triangles [АМГ, ВМГ], parts of the triangle $[\mathrm{AB} \Gamma]$. They concluded that the triangle $\mathrm{AB} \Gamma$ is right, as they identify in its form a theorem they know (if the median length of a triangle side equals half the length of that side, the triangle is right).

S2: Like a triangle with a right angle... the small bar is its median.

$\mathrm{S} 1$ : A right-angled triangle [she shows $\mathrm{AB} \Gamma]$... with this angle [she shows $\Gamma$ ] being right.... Because we know that this is median [she shows $\Gamma \mathrm{M}$ ] with this one as the hypotenuse [she shows $\mathrm{AB}$ ], which we know equals with that half...there see it? ... this is the right angle [she shows $\Gamma]$... it's the median theorem, remember it? ... so, it always has a right angle.

S2: So, a setsquare, we can use it for right angles (table 5).

To the students, it seems that the artifact embodies the triangle median theorem, and the assembly of the artifact is enough for them to deem the angle as right. In fact, they are proving the reverse of the theorem, based exclusively on the artifact assembly.

TABLE 5

EPISODE 2: CONSTRUCTION OF RIGHT ANGLES THE ARTIFACT EMBODIES A GEOMETRICAL THEOREM

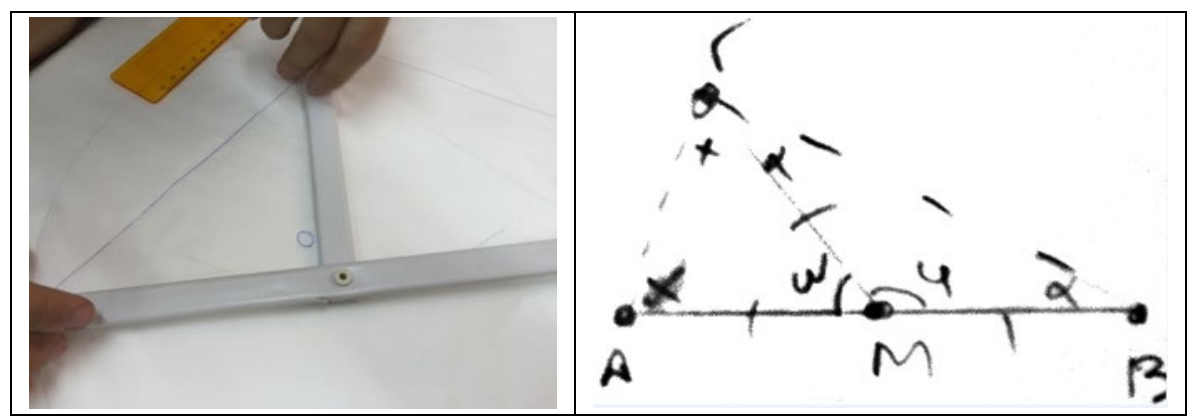


The boys' team drew a straight line and placed on it the free end [B] of the small rod [BK], which they stabilized with a nail on a plastic platform, and one of the free ends $[\Gamma]$ of the large rod $[А \Gamma]$. They shaped the form of the artifact with a triangle $\mathrm{AB}$. The students, based on their drawing, decided to search for the type of the triangle:

S4: In this way we can make a triangle .... In which this is the median [he shows BK] ... it looks like a right-angled triangle.

S5: A right-angled triangle .... but, how can we know for sure that this angle is a right one? [he means the angle B]

Using propositions related to the angles of a triangle (the base angles of an isosceles triangle are equal and the sum of the angles is 1800) and applying algebraic techniques, they concluded that the triangle is right-angled.

S3: Look, these angles ( $\omega$ and $\omega)$ are equal and these are equal ( $\varphi$ and $\varphi$ ); we add them $(\omega+\omega+\varphi+\varphi)$ and they are equal to 180 degrees, we divide them by 2 and we have 90 degrees $(\omega+\varphi)$.

S4: This is the median with the hypotenuse... which we know that is equal to this half...here, you see... this is a right triangle...this is a right angle...remember?

S5: Yes, the reverse of the theorem that in a right-angled triangle the median is the half of the hypotenuse. Because $\mathrm{BK}=\mathrm{KA}=\mathrm{K} \Gamma$, the triangle is a right one (table 6).

TABLE 6

EPISODE 2: CONSTRUCTION OF RIGHT ANGLES ARTIFACT EMBODIES A GEOMETRICAL THEOREM

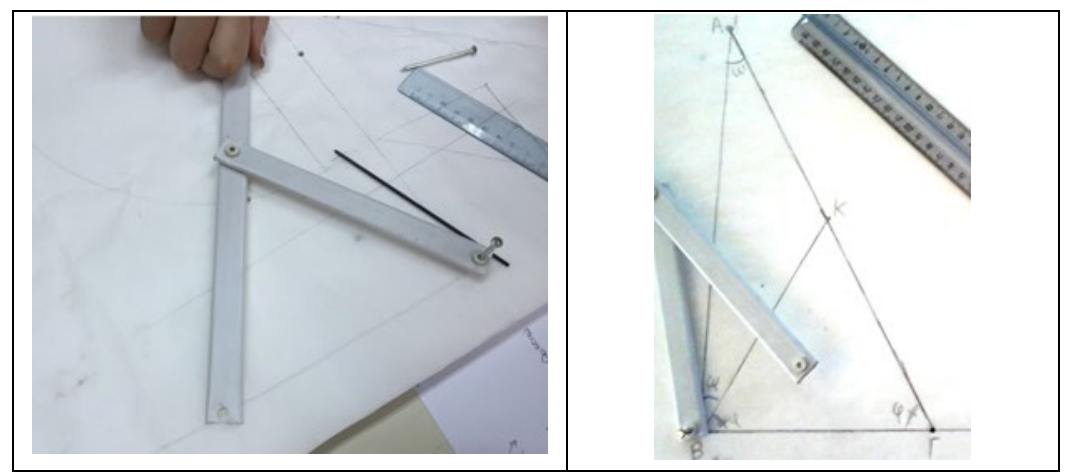

Episode 3- Construction of Perpendicular Lines

The conclusion that the artifact integrates a right angle led the group of boys to attempt to construct two perpendicular lines, using the artifact.

S4: The tool has always a right angle.

T: What could you construct with the tool?

S4: Any shape with a right angle. 
S5: Could we construct perpendicular lines?

\section{T: Try it.}

Holding a free end $[\mathrm{B}]$ of the small rod $[\mathrm{BK}]$ stable, dragging one of the free ends $[\Gamma]$ of the large rod $[\mathrm{A} \Gamma]$ alongside a straight line and placing pencils at the hinge $[\mathrm{K}]$ and the third free end $[\mathrm{A}]$ of the large rod, they concluded that "this $(\mathrm{K})$ gives a circle, no, ... semicircle, no, ...quadrant and this (he points at A) gives the perpendicular line. (S3)" The figure produced by the pencil was not complete and did not resemble a straight line. The students using the reductio ad absurdum method claim that the straight line AB that is drawn by the $\mathrm{A}$ of the rod $\mathrm{A} \Gamma$ is a perpendicular line "we proved for the right angle $[\mathrm{AB} Г]$ without the theorem'.

S3: It is enough to show that for another position [of the large rod $\mathrm{A} \Gamma]$... that for this point [A] and for that point [ $\left.\mathrm{A}^{\prime}\right]$, the two parts [he points at $\mathrm{AB}$ and $\left.\mathrm{A}^{\prime} \mathrm{B}\right]$ are perpendicular on a line at the point $\mathrm{B} \ldots$ we will write these relationships twice [he refers to the sum of the angles of the triangle being 1800] for each of the isosceles triangles [he means the triangles $\mathrm{AB} \Gamma$ and $\left.\mathrm{A}^{\prime} \mathrm{B} \Gamma^{\prime}\right]$.

From the moment that students proved that they can construct perpendicular lines (table 7), they concluded that they can also construct parallel lines as perpendicular to the same line and also rectangles and squares.

TABLE 7

\section{EPISODE 3: CONSTRUCTION OF PERPENDICULAR LINES}

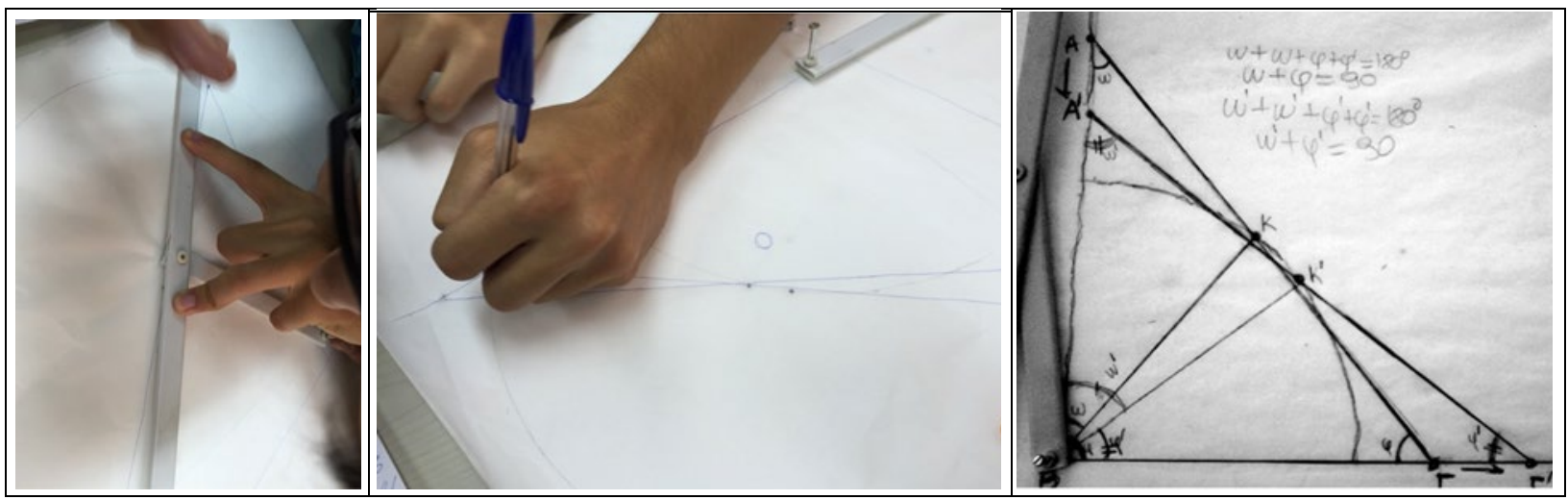

\section{CONCLUSION}

In this paper, we discuss the results of a teaching experiment in the integration of an artifact in teaching Euclidean geometry at upper secondary school. We focused a) on the exploration processes followed by the students in order to discover the mathematics that were integrated by the introduction of an artifact and b) on the potentialities of the artifact as a geometrical tool on students with no prior experience with other tools apart from the pair of compasses and rulers. As demonstrated from the description of the data of the first unit, students of both subgroups succeeded in discovering the mathematics incorporated in the structure and form of the physical model of that particular artifact. As demonstrated from episodes 1,2,3 of the second unit, both boys and girls, through working with the artifact, discovered its uses, as a pair of compasses and a set-square for geometrical constructions and identified in the artifact the incorporation of theorems and their reverses as well as developed interesting strategies of argumentation and proof. The students in episode 1, although they developed different investigatory strategies of what the potential of the linkage is, identified the artifact, via its use, as a pair of compasses by performing position combinations between nails 
and a fixed-point and they developed interesting strategies of argumentation. In episode 2, the group of girls identifies in the artifact the integration of known theorems as well as their reverse.

On the contrary, for the team of boys, the use of the artifact as a tool with a perpendicular angle, was not enough for them to justify its use as a set square in other geometrical constructions like the one developed in episode 3. Their inaccurate drawing led them to prove their claims "without the theorem" but via correlating known theories and the reduction ad absurdum method.

Direct contact of the students with the artifact seemed to act as motive force to connect existing geometry knowledge with the mathematics incorporated by the artifact. The artifact limitations did not prevent students from transforming it into an object of knowledge. Although drawings produced by the tracing-point were inaccurate and only presented part of the trajectory, those drawings seem to be able to activate mental experiments, overcoming of limitations, and deduction of their generalizations. The students are led towards proposing their proof by taking advantage of the characteristics of the models of the artifact they are offered.

\section{REFERENCES}

Bussi, M.B., \& Mariotti, M.A. (2008). Semiotic mediation in the mathematics classroom: Artifacts and signs after a Vygotskian perspective. Handbook of International Research in Mathematics Education, p.746.

Bussi, M.G.B., Taimina, D., \& Isoda, M. (2010). Concrete models and dynamic instruments as early technology tools in classrooms at the dawn of ICMI: From Felix Klein to present applications in mathematics classrooms in different parts of the world. ZDM, 42(1), 19-31.

Fujita, T., Jones, K., \& Kunimune, S. (2010). Students' geometrical constructions and proving activities: A case of cognitive unity. In Proceedings of the 34th Annual Conference of the International Group for the Psychology of Mathematics Education (Vol. 3, pp. 9-16).

Mariotti, M.A. (2002). Influence of technology advances on students' math learning. Handbook of International Research in Mathematics Education. Mahwah, Lawrence Erlbaum.

Mariotti, M.A. (2013). Introducing students to geometric theorems: How the teacher can exploit the semiotic potential of a DGS. ZDM, 45(3), 441-452.

Martignone, F. (2011). Tasks for teachers in mathematics laboratory activities: A case study. In Proceedings of the 35th Conference of the International Group for the Psychology of Mathematics Education (Vol. 3, pp. 193-200).

Steffe, L.P., \& Thompson, P.W., \& Von Glasersfeld, E. (2000). Teaching experiment methodology: Underlying principles and essential elements. Handbook of Research Design in Mathematics and Science Education, pp. 267-306.

Trninic, D., Reinholz, D., Howison, M., \& Abrahamson, D. (2010). Design as an object-to-think-with: Semiotic potential emerges through collaborative reflective conversation with material. In Proceedings of the thirty-second annual meeting of the North-American chapter of the international group for the psychology of mathematics education (PME-NA 32) (Vol. 6, pp. 1523-1530).

Trninic, D., \& Abrahamson, D. (2012). Embodied artifacts and conceptual performances. In Proceedings of the International Conference of the Learning Sciences: Future of learning (ICLS 2012) (Vol. 1, pp. 283-290).

Vérillon, P., \& Andreucci, C. (2006). Artefacts and cognitive development: How do psychogenetic theories of intelligence help in understanding the influence of technical environments on the development of thought. International Handbook of Technology Education, pp. 399-416. Brill Sense.

Verillon, P., \& Rabardel, P. (1995). Cognition and artifacts: A contribution to the study of though in relation to instrumented activity. European Journal of Psychology of Education, pp. 77-101. 